\title{
Retarded Field Energy Analyzer as a tool to Study the Ion Velocity Distribution Functions on Radio Frequency Argon Plasma in Expanding Magnetic Field at Low Pressures
}

\author{
L. N. Mishra \\ Department of Physics, Patan Multiple Campus, Tribhuvan University \\ Patangate, Laltipur, Nepal. \\ Correspondence to: 1misira@gmail.com
}

\begin{abstract}
Plasma expanding in the space along the magnetic filed is well known phenomenon. This plasma device was constructed to investigate the space plasma in laboratory in connection with plasma flows, electron distribution, ion distribution, instability and turbulence. For this purpose, the low-temperature plasma is produced by means of a $13.56 \mathrm{MHz}$ Helicon plasma source at 300-1000 Wrfpower. The plasma is expanding from the $13.5 \mathrm{~cm}$ diameter source into a $150 \mathrm{~cm}$ long chamber of $60 \mathrm{~cm}$ diameter. Ion energy and its velocity distribution produced by a current-free double layer at the expansion region have been studied by means of retarding field energy analyzers. Furthermore, the effects due to the geometrical shape of the expanding magnetic field in plasma flows have also been investigated.
\end{abstract}

Keywords: electron distribution, ion distribution, helicon plasma and turbulence.

\section{Introduction}

It is well-known that the velocity distributions of ions and electrons play the crucial role in space plasma as well as plasma processing to the surface modifications of materials for a wide variety of industrial applications. In the former case, ions collide much more frequently with the neutral molecules than with other ions at auroral latitudes. These phenomena could be carried out by taking the information on the distribution either from high resolution ground-based optical measurements or from retarding potential analyzers carried on rockets and satellites. But, in the later case, the microelectronic industry use plasma discharges for the processing of semiconductor wafers. So, the mechanism or dynamics of ions or electron interact to the semiconductors surface due to their bombardment is quite important where they interchange their energies. However, the ion current flux and ion energy determine the effectiveness of plasma process.

The rf source is commonly used for plasma processing for this application. In this source, applied power is initially absorbed by electron and then transferred to the neutral species and ions by collisions. Moreover, besides the interaction between rf field and electrons there might be the interaction between ions and $\mathrm{rf}$ field as well. In the case of inductive plasma sources, an axial magnetic field with sinusoidal time dependence exists due to the current flowing through the inductive-source solenoid. This magnetic field induces an electric field by Faraday's law, 


$$
\frac{\partial \vec{B}}{\partial t}=-\nabla \times \vec{E}
$$

The resulting electric field is in the azimuthal direction, sinusoidally varying in time as the magnetic field, but out of phase by $\pi / 2 \mathrm{rad}$. This electric field accelerates the ions normal to the coil axis, which results in a higher effective temperature in that direction as explained by the following equation.

$$
f(v)=\frac{\omega}{2 \pi} \int_{0}^{2 \pi / \omega} f(v, t) d
$$

where $\omega$ is the RF angular frequency and $f(v$, $t)$ is the instantaneous ion velocity distribution. The effective temperature measured from $f(v)$ is a superposition of the true thermodynamic temperature of $f(v, t)$ and coherent motion of ions in the RF field. The cyclotron effects were neglected due to the small background magnetic field.

The laser induced fluorescence (LIF) Scime et al., 2000, Sadeghi et al 1991, Biloiu et al 2008, Keese et al 2007, Zintl et al 1994, is quite popular to measure the ion velocity distribution (IVDF) in rf plasmas though its first application to plasma diagnostics by Stern and Johnson Stern et al three decades ago. Besides this, retarding field energy analyzers (RFEAs) have been used extensively for determining the IED in plasma discharges in recent decades Coburn et al 1972, Kuypers et al 1988 and 1990,Ingram et al 1990, Janes et al 1992, Charles et al 1992 and 1993. The potential of a grid placed in front of a beam of ions is increased until the beam is stopped. By collecting and measuring the current of ions passing the grid, as a function of the grid potential, the integrated form of the IED is obtained. The true distribution of ion energies is obtained by differentiating this current potential characteristic. The analyzer can be mounted at either the grounded or the rf biased electrode when investigating parallel plate reactors.

In this paper, the ion velocity distribution functions obtained by RFEA in the down stream region of rf source is reported. Measurements carried out in different guiding coil currents to study the effect of magnetic field on the measured IVDFs.

\section{Experimental Set up}

The experimental arrangement for the characterization of argon rf plasma in the Njord device is shown as in Fig. 1. It consists of a source $(\phi 21 \mathrm{~cm} \times 31.3 \mathrm{~cm})$ attached to a vacuum chamber made of stainless steel, called the diffusion chamber $(\phi 60 \mathrm{~cm} \times 120 \mathrm{~cm})$. More specifically, plasma is formed in a Pyrex tube source $(\phi 13.8 \mathrm{~cm} \times 30.5 \mathrm{~cm})$ inside the source chamber. The source and diffusion chambers are connected at a cylindrical port $(\phi 20 \mathrm{~cm} \times$ $6 \mathrm{~cm}$ ) called source tube extension. The plasma source is adapted from the similar source of the "Chi-Kung" at Australian National University. It consists of a double -saddle rf-antenna fed with $13.56 \mathrm{MHz} \mathrm{CW}$ from a Henery $8 \mathrm{~K}$ Ultra rf amplifier and is fitted with a pi-network tuning system with vacuum capacitors. The rf power applied for the breakdown of the gas is in the range 200-600 W.

A weak magnetic field of maximum $15 \mathrm{mT}$ is applied in the source region by means of two coils outside the saddle-type helicon antenna placed around the Pyrex tube source chamber. In addition, third coil called guiding coil is placed $58 \mathrm{~cm}$ downstream from the end plate of the source, which defines the origo along the axis of the source. The field produced by the latter coil could be varied from 0 to $15 \mathrm{mT}$, 

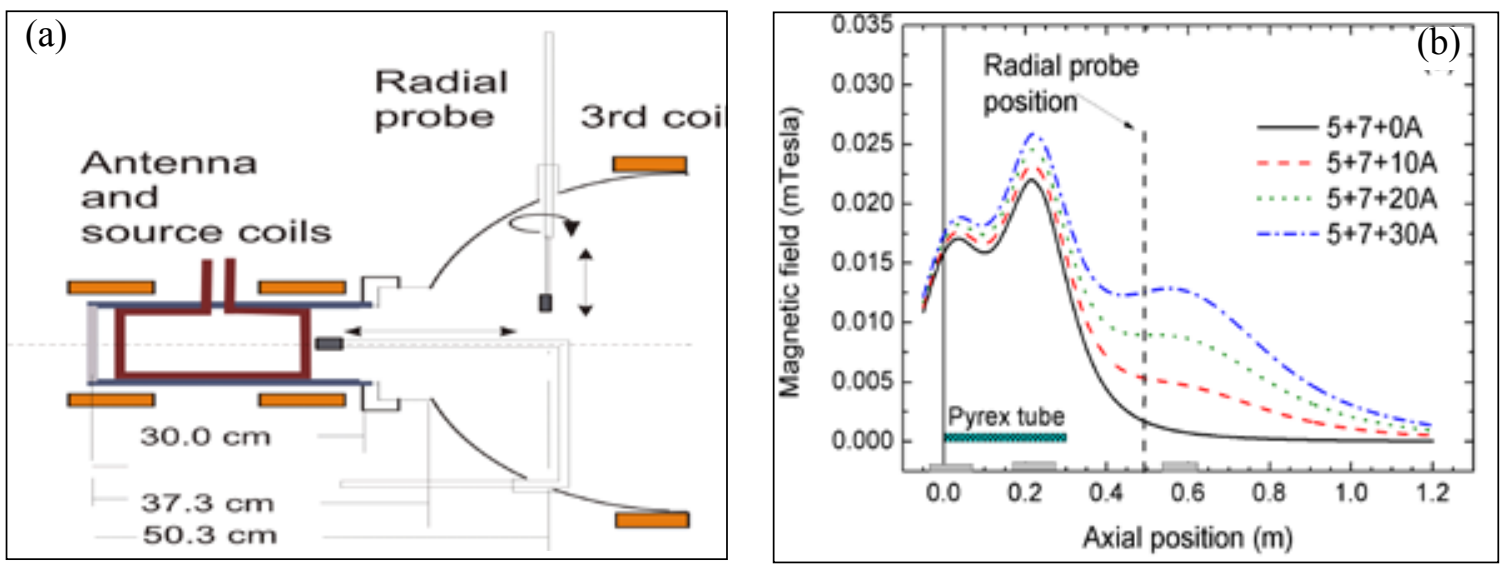

Figure 1. Experimental arrangement : RF source with probe (a) and magnetic field magnetic field configuration (b).

so that an inflection point is produced between the second and third coil already at fields of less than $5 \mathrm{mT}$ that of third coil, as indicated in figures (1b) and (1c). The vacuum system in the working chambers consist of one turbomolecular pump backed by one $350 \mathrm{l} / \mathrm{min}$ rotary pump, with which the base pressure of the system is kept below 0.02 mTorr. Its operating pressure is $\leq 0.18$ mTorr. The flow control is carried out by a Omega FMA 5400/5500 series flow controller with maximum capacity 20 $\mathrm{cm}^{3} \mathrm{~min}^{-1}$. This controller is driven with remote computer system.

A retarding field energy analyzers (RFEAs) shown in Fig. 2 is inserted through ports in a radial direction in the ion diffusion region of low pressure argon plasma typically at $0.18 \mathrm{~m}$ Torr. The latter RFEA could be rotated thoroughly $360^{\circ}$ around its axis. This RFEA had orifice diameter $1 \mathrm{~mm}$, and is constructed with a mesh across the orifice in the grounded front end plate. The electron repeller grid was biased at $-90 \mathrm{~V}$, the discriminator grid had variable bias from $-100 \mathrm{~V}$ to $+100 \mathrm{~V}$, the secondary repeller grid was biased at $-20 \mathrm{~V}$ and the collector at -9 $\mathrm{V}$. The overall system length was $2 \mathrm{~mm}$ with
$0.50 \mathrm{~mm}$ space between the grids. There is good electrical connection between the plate and the grounded analyzer housing. The discriminator was biased in 500 steps per scan from $-100 \mathrm{~V}$ to $+100 \mathrm{~V}$. At each step the collector current, measured over a $32 \mathrm{k} \Omega$ resistor, was digitized into 500 samples which were then averaged into one single value, and written to file for further analysis.

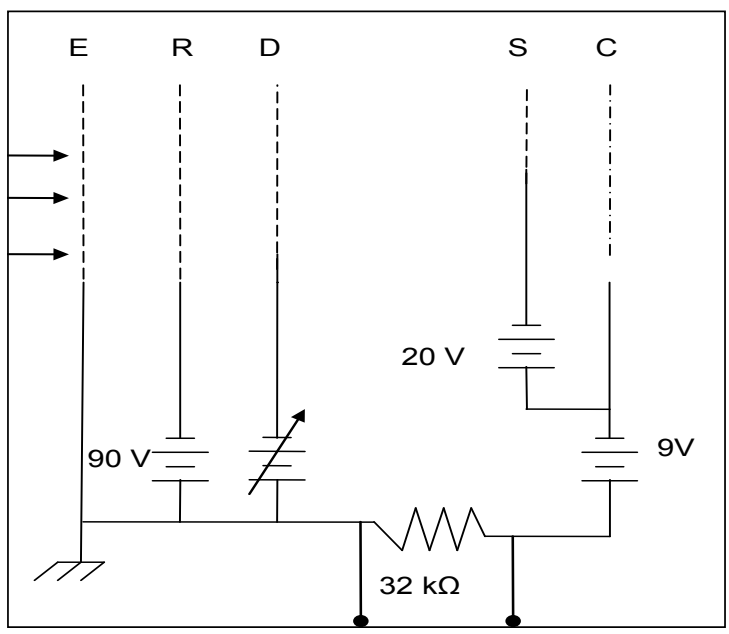

Figure 2. RFEA bias circuit: $\mathrm{E}$ - earthed, R repeller, $\mathrm{D}$ - discriminator, $\mathrm{S}$ - secondary emission suppressor, $\mathrm{C}-$ collector. 


\section{Results and Discussions}

The key parameters of background rf plasma are expressed in terms of plasma potential, electron temperatures and plasma density. In this case, a compensated Langmuir probe of dimension $0.25 \mathrm{~mm}$ diameter and $9.0 \mathrm{~mm}$ in length was employed to measure those above mentioned parameters.

Figure 3(a) and 3 (b) show the variation of upstream and downstream ion saturation current at various currents in the guiding coil obtained with a RFEA keeping gas flow at 1.5 sccm and $1.2 \mathrm{sccm}$ with forward power at 600 $\mathrm{W}$. It is found that when RFEA facing towards the source the upstream ion saturation current increases as guiding coil current gradually increases up to $15 \mathrm{~A}$ after then they begin to decrease as guiding coil currents increase further more. But, in the case of downstream ion saturation current, it increases up to $15 \mathrm{~A}$ and then slowly decreases. The upstream ion saturation current is larger than down stream ion saturation currents infers the flow of ions from upstream to down stream direction. Actually, it does not give the actual flow measurements because the RFEA itself a large grounded object which creates a wake. The direct estimate of the flow velocity can be measured by Mach probe.

Figures 4 (a) and (b) show the typical characteristics curves of collector current versus discriminator voltage and the corresponding derivative representing the ion energy distribution function (IEDF) at different angle of orientation obtained with the radial analyzer placed at $\mathrm{z}=50.5 \mathrm{~cm}$ and $\mathrm{r}=0 \mathrm{~cm}$ with a continuous argon plasma operating at $600 \mathrm{~W}$ rf power and 0.24 mTorr pressure. The three measurements obtained with the analyzer at $0^{0}$ (facing toward source), $90^{\circ}$ (facing side walls) and $180^{\circ}$ (facing opposite the source) infer that one peak distribution at $90^{\circ}$ (dot line), $180^{\circ}$ (dash line) and two significant peaks corresponding to plasma potential and an ion beam potential at $0^{0}$ (solid line) with rotation of axis of analyzer. The existence of the two peaks viz. one plasma potential and another beam potential, is the evidence of the formation of double layers. Typically, it is found that local plasma potential

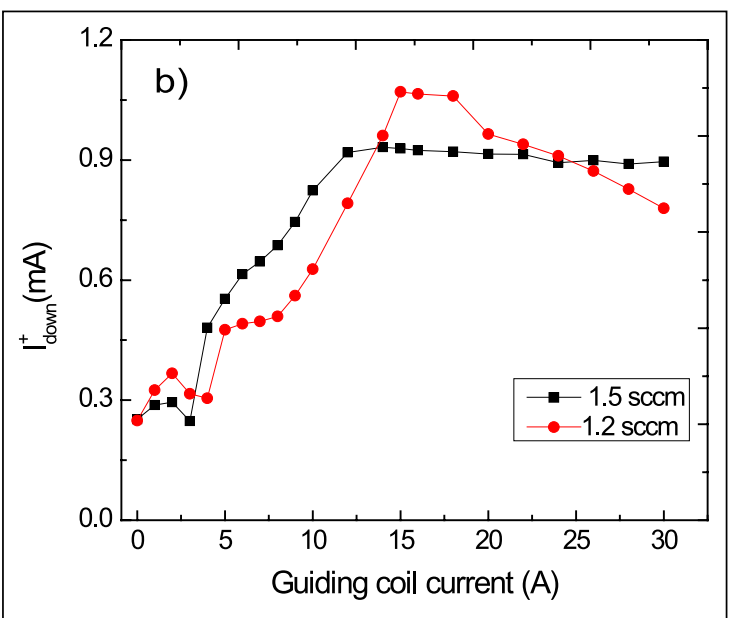

Figure 3. Typical profiles of upstream ion saturation current (a) and downstream ion saturation current (b) obtained with RFEA facing the source and opposite side of the source at different gas flow viz. square for $1.5 \mathrm{sccm}$, circle for $1.2 \mathrm{sccm}$. 
which is due to the background ion population as $50 \mathrm{~V}$ and that of beam potential as $77 \mathrm{~V}$. Consequently, one can be estimated the double layer potential drop as $\phi_{\mathrm{dl}}=\mathrm{V}_{\text {beam }}-\mathrm{V}_{\mathrm{p}}=27 \mathrm{~V}$. In more detail, it could be found in Byhring et al. [2008] and Fredriksen et al. [2010].

Figure 5 shows the typical profiles of plasma potentials as function of guiding coil current obtained with RFEA facing towards the side wall. It is seen that the plasma potential gradually increases as the guiding coil current increases. It also found that plasma potential decreases as the gas flow increases. In this case, RFEA is kept at sidewall direction that is the analyzer placed $90^{\circ}$ with axis of rotation to obtain the plasma potential. It infers from the Fig. 1 (b) and Fig. (5) that at the zero or low magnetic field maintaining $0 \mathrm{~A}$ with the help of current providing in third coil, a large number of plasma particles diffuse to the walls which enhance the reduction of background plasma. But, when the magnetic field increases by changing the current from 10 to $20 \mathrm{~A}$, all the fluxes are staying within the cross-section of port - dome intersections which enhances the increment of plasma potential. After $25 \mathrm{~A}$ the fluxes of plasma particles try to deviate outwards from the cross-section of port-dome intersection due to expanding magnetic field. Hence, the plasma potential will remains almost constant beyond $25 \mathrm{~A}$ coil current.

Figure 6 shows the typical profiles of electron temperature and plasma density obtained with compensated Langmuir probe keeping gas flow $1.5 \mathrm{sccm}$ and input power $600 \mathrm{~W}$. It is found that plasma density increases gradually and will attain optimum when guiding coil current will be $15 \mathrm{~A}$. It will gradually decrease when guiding coil current increases further more. But, in the case of electron temperature, there

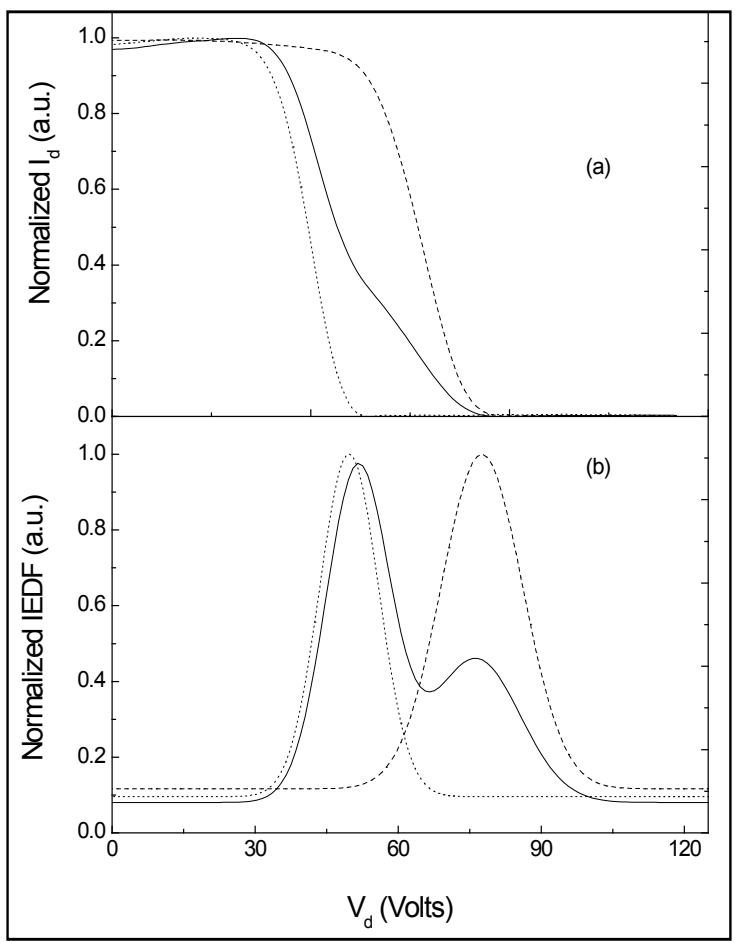

Figure 4. Normalized collector current (a) and IEDF (b) versus discriminator voltage obtained with RFEA located at $\mathrm{r}=0 \mathrm{~cm}$ for various collecting angles (solid line) $\alpha=00$ (radial measurements with RFEA facing towards the source), ( dot line) $\alpha=900$ (radial measurements with RFEA facing side walls of the chamber), (dash line) $\alpha=1800$ (radial measurements with RFEA facing opposite the source).

is almost same while the guiding coil current increases.

Figure 7 (a) and 7 (b) show the typical evolutions of ion velocity distribution as function of ion speed. These distributions are obtained with the conversion of the first derivative of $\mathrm{I}-\mathrm{V}$ characteristics according to equation [22]

$$
f(v)=\frac{m_{i}}{A T^{4} e^{2}}\left(-\frac{d I}{d V}\right)
$$

where $\mathrm{T}$ is the transmission coefficient for the grid $(=0.4)$, A the area of RFEA $\left(=3.12 \times 10^{-6}\right.$ 


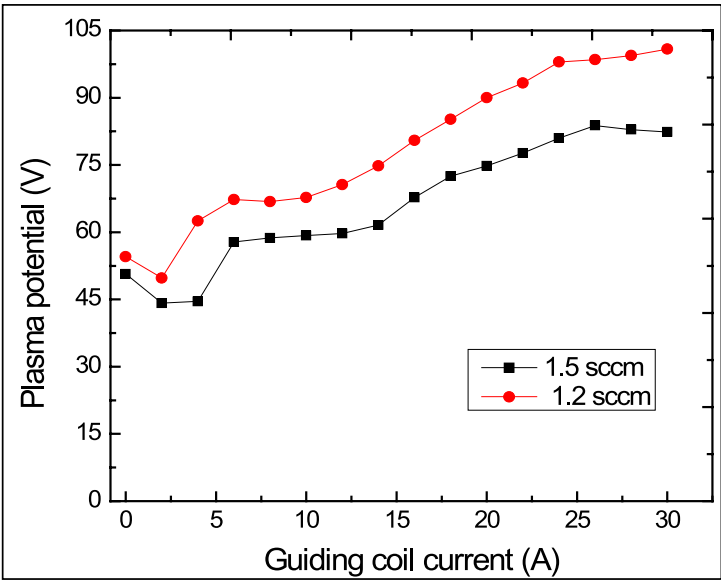

Figure 5. A typical profile of plasma potential as a function of guiding coil current.

$\mathrm{m}^{-2}, \mathrm{~m}_{\mathrm{i}}$ the mass of ion $\left(=40 \times 1.67 \times 10^{-27} \mathrm{~kg}\right.$ for Argon) and e the charge of electron (= 1.6 $\mathrm{x} 10^{-19} \mathrm{c}$ ). It is clearly seen that the ion is highly populated when its velocity becomes 17000 $\mathrm{m} / \mathrm{s}$ for $1.2 \mathrm{sccm}$ flow and while that for 1.5 $\mathrm{sccm}$, its velocity will be $16000 \mathrm{~m} / \mathrm{s}$.

Figure 8 (a) and 8 (b) show the typical evolutions of ion velocity distribution as

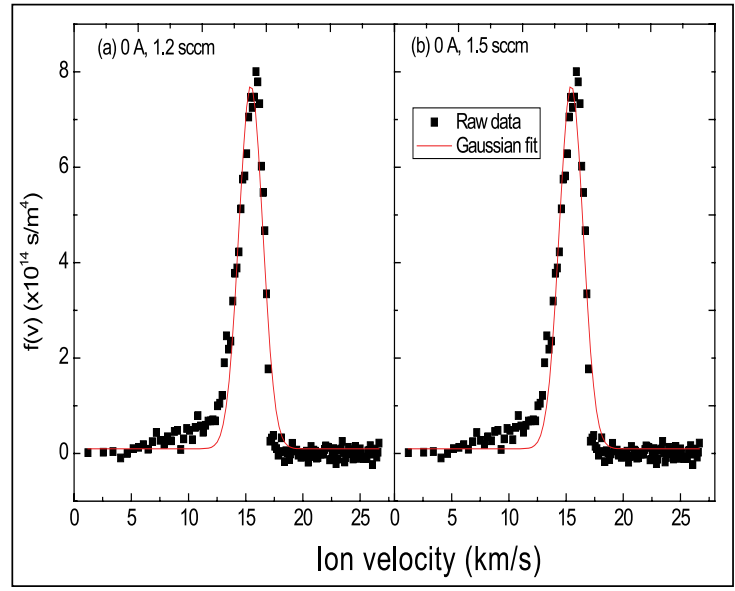

Figure 7. Typical ion velocity distribution at guiding coil current 0 A keeping pressure 0.24 mTorr and power $600 \mathrm{~W}$.

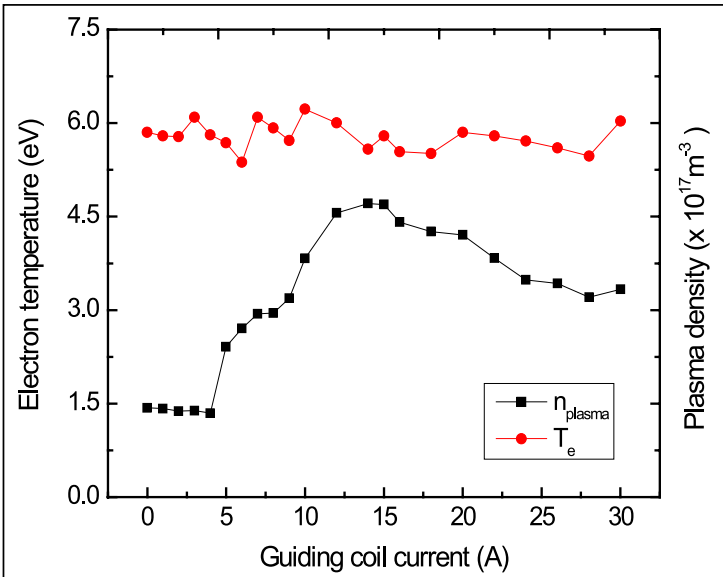

Figure 6. A typical electron temperature and density profiles as a function of guiding coil current.

function of ion speed. It is clearly seen that the ion begins to highly populate when its velocity becomes $20000 \mathrm{~m} / \mathrm{s}$ for 1.2 sccm flow and while that for $1.5 \mathrm{sccm}$, its velocity will be $18000 \mathrm{~m} / \mathrm{s}$. It infers from Fig. 7 and Fig. 8 that population of ion acceleration increases as the guiding coil current increases from $0 \mathrm{~A}$ to $15 \mathrm{~A}$ which could be seen in density profiles in Fig. 4.

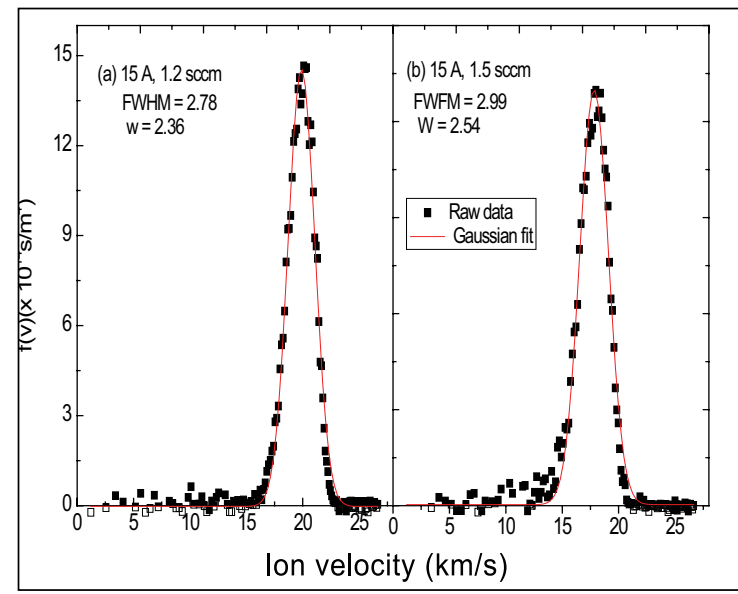

Figure 8. Typical ion velocity distributions at guiding coil current 15 A keeping pressure 0.24 mTorr and power $600 \mathrm{~W}$. 


\section{Conclusions}

The background plasma properties in terms of ion density, plasma potential, electron temperature and plasma density have characterized in a Njord device. These plasma parameters are also studied in the configuration of different magnetic field by providing the different current in guiding coil. It is found that plasma potential increases as guiding coil increases in third coil. Plasma potential gradually increases and it will attain the maximum value when guiding coil current will be at 14 to $15 \mathrm{~A}$ and then gradually decreases as the guiding coil current decreases. It is also noticed that the shape of magnetic field alters when third coil current is varied from $0 \mathrm{~A}$ to 30 A. It is also seen that the ion begins to highly populate when its velocity becomes $20000 \mathrm{~m} / \mathrm{s}$ for $1.2 \mathrm{sccm}$ flow and while that for $1.5 \mathrm{sccm}$, its velocity will be $18000 \mathrm{~m} / \mathrm{s}$.

\section{Acknowledgments}

This work was supported by Norwegian Research Council. The author is grateful to Professor Åshild Fredriksen for invaluable support. Editor N. P. Chapagain thanks the reviewers for their assistance in evaluating this paper.

\section{References}

Biloiu C, E. Scime, X. Sun and B. McGeehan (2008), Temporal evolution of bimodal argonion velocity distribution in an expanding helicon plasma, IEEE Transactions on Plasma Science 36, 1376.

Byhring H. S., Å Fredriksen, C. Charles and R. W. Boswell (2008), Double layer in an expanding plasma: Simultaneous upstream and downstream measurements, Phys. Plasmas, 15, 102113.

Charles C. (1993), Measured ion energy distribution functions in low pressure argon plasma produced by a Helicon sorce, J. Vac. Sci. Technol. A 11, 157.

Charles C., R. W. Boswell, and R. K. Porteous (1992), Measurement and modeling of ion energy distribution function in a low pressure argon plasma by $56 \mathrm{MHz}$ helicon source, J. Vac. Sci. Technol. A 10,398.

Coburn J. W. and E. Kay (1972), Ion transport in an electron cyclotron resonance plasma J. Appl. Phys. 43, 4965.

Fredriksen $\AA$, L. N. Mishra and H. S. Byhring (2010), The effect of downstream magnetic field on current free doble layers and beam formation in the Njord helicon plasma device, Plasma Sources Sci. Technol. 19, 034009.

Janes J. and C. Huth (1992), Bombardment energies of oxygen in low pressure reactive ion etching, Appl. Phys. Lett. 61, 261.

Keesee A. M. and E. E. Scime (2007), Neutral density profiles in argon helicon plasmas, Plasma Sources Sci. Technol. 16, 742-749.

Kuypers A. D. and H. J. Hopman (1988), Ion energy measurement at the powered electrode in an rf discharge, J. Appl. Phys. 63.

Kuypers A. D. and H. J. Hopman, (1990), Ion energy measurement at the powered electrode in variable magnetic field, J. Appl. Phys. 67, 1229.

Ingram S. G and N. S. J. Braithwaite (1990), rf modulation of positive-ion energies in low pressure, J. Appl. Phys. 68, 5519.

Sagdeghi N, T. Nakono, D.J. Trevor and R. Gottscho (1991), Ion transport in an electron cyclotron resonance plasma, Appl. Phys. 70, 2552.

Scime E E et al. (2000), Phys. Plasmas 7, 2157.

Stern R. A. and J. A. Johnson (1975), Plasma ion diagnostics using resonant fluorescence,. Phys. Rev. Lett. 34, 1548.

Zintl M and R. McWilliams (1994), Improved optical tomography device, Rev. Sci. Instrum. $\mathbf{6 5}, 2574$ 